\title{
A Discussion on Contact Condition in Simulation of RCFT Columns
}

\author{
Alifujiang XIAMUXI ${ }^{1, a, ~}{ }^{*}$, Akenjiang TUOHUTI ${ }^{1, b}$, Akira HASEGAWA ${ }^{2, c}$ \\ ${ }^{1}$ College of Architecture and Civil Engineering, Xinjiang University, Urumqi 830047, China \\ ${ }^{2}$ Department of Environmental and Civil Engineering, Hachinohe Institute of Technology, \\ Hachinohe, Japan \\ ailikyurt@xju.edu.cn, ${ }^{b}$ akjt@xju.edu.cn, chasegawa@hi-tech.ac.jp \\ ${ }^{*}$ Corresponding author
}

Keywords: Numerical Simulation, Contact, CFT, RCFT, ADINA.

\begin{abstract}
In numerical simulation of Concrete Filled Steel Tubular (CFT) columns the treatments to simulation the contact between steel tube and concrete differed greatly between the researchers, and there is also no any unified standard for the friction between the concrete and steel materials. To examine proper contact condition in simulating Reinforced Concrete Filled Steel Tubular (RCFT) columns with ADINA, numerical simulations are performed employing frictionless, frictional and bonding contact conditions between steel tube and concrete core, the analytical results are compared against experimental results, and the applicability of each contact on RCFT is observed, and a proper contact condition is proposed.
\end{abstract}

\section{Introduction}

The treatments in simulating the contact between steel tube and concrete in numerical analysis of Concrete Filled Steel Tubular structure (CFT) vary between the researchers. There have been examples of bond, frictional and frictionless contact models. Choi et al. [1] adopted frictionless contact condition in CFT columns under axial compression with ADINA. Hu et al. [2] modeled the contact in CFT columns subjected to axial compression with infinitesimal sliding and friction between the concrete and the steel tube. The contact surfaces between the concrete and the steel tube are allowed to separate but not to penetrate each other. The friction coefficient used in their analyses was 0.25 . Li et al. [3] simulated the contact in concrete filled double skin tubular columns under axial compression using a Coulomb friction model in the tangential direction between surfaces of steel tube and concrete core with a friction coefficient of 0.6. Han et al. [4] employed Coulomb friction theory with a friction coefficient of 0.6 in contact modeling of CFT subject to torsion. Zeng et al. [5] employed Coulomb friction theory with a friction coefficient of 0.6 in simulating the contact between stiches and concrete. Hou et al. [6] employed Coulomb friction theory with a friction coefficient of 0.6 in contact modeling of CFT subject to impact force. Mao et al. [7] assumed complete bond between steel tube and concrete in modeling the steel-concrete composite beam joints. Kwak et al. [8] simulated cyclic behavior of CFT columns with bonded contact condition. All of those indicate the treatments of contact between steel tube and concrete differed greatly between the researchers. There is also no any unified standard for the friction between the concrete and steel materials as well.

To examine the contact condition in simulating the Reinforced Concrete Filled Steel Tubular structure (RCFT) with ADINA, in this study, simulations are performed with above frictionless, frictional and bonding contact conditions, the analytical results are compared against experimental results, and the applicability of each contact on RCFT is discussed.

\section{Experimental Results}

The experimental results on RCFT by Suzuki [9] were used as a comparison standard for the simulation. Three RCFT columns were selected with three different steel tube thicknesses. The columns are labeled with a prefix " $T$ " which implies the first capital letter of "Thickness of steel 
tube" and the two digits of number which implies the thickness of steel tube after removing decimal point ".". The experimental parameters for these selected columns are listed in Table1.

Tab. 1 Parameters of RCFT Columns

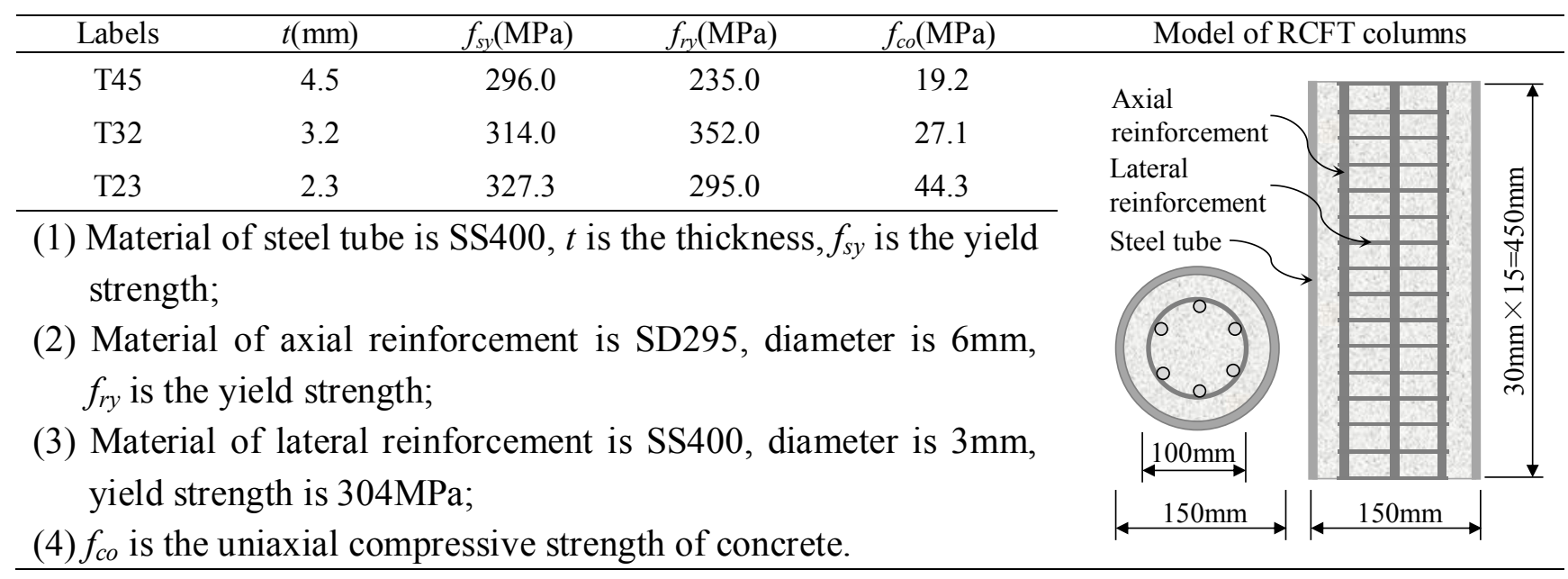

Tab. 2 Modeling Parameters for Contact

\begin{tabular}{|c|c|c|c|c|}
\hline \multicolumn{3}{|c|}{ RCFT Columns and Labels } & \multirow{2}{*}{ Coefficient of friction $(\mu)$} & \multirow{2}{*}{ Contact conditions } \\
\hline $\mathrm{T} 45$ & $\mathrm{~T} 32$ & $\mathrm{~T} 23$ & & \\
\hline T45-F00 & T32-F00 & T23-F00 & 0 & Frictionless \\
\hline T45-F02 & T32-F02 & T23-F02 & 0.2 & Frictional \\
\hline T45-F05 & T32-F05 & T23-F05 & 0.6 & Frictional \\
\hline T45-F08 & T32-F08 & T23-F08 & 0.8 & Frictional \\
\hline T45-FB & T32-FB & $\mathrm{T} 23-\mathrm{FB}$ & - & Bonded \\
\hline $\mathrm{T} 45-\mathrm{T}$ & $\mathrm{T} 32-\mathrm{T}$ & $\mathrm{T} 23-\mathrm{T}$ & - & Test \\
\hline
\end{tabular}

\section{Numerical Simulation}

\section{Contact Model}

A constraint-function model built in ADINA is employed to simulate the contact condition between the steel tube and in-filled RC in RCFT.

When two surfaces come into contact with each other, normal contact force $\lambda$ acts on the contact surfaces (see Fig.1). The normal contact conditions can ideally be expressed as:

$$
g \geq 0 ; \quad \lambda \geq 0 ; \quad g \lambda \geq 0
$$

Where $g$ is a gap. The inequality constraints are replaced by:

$$
w(g, \lambda)=\frac{g+\lambda}{2}-\sqrt{\left(\frac{g+\lambda}{2}\right)^{2}+\varepsilon_{N}}=0
$$

Where $\varepsilon_{N}$ is a small user-defined parameter. The function is shown in Fig.1. Forces on contact surface Fig.2. It involves no inequalities, and is smooth and differentiable. The parameter $\varepsilon_{N}$ is set to $1.0 \times 10^{-12}$ which is suitable for most applications and should rarely be modified.

When two surfaces come into contact with each other, frictional force $F_{T}$ occurs in the direction tangential to the contact surfaces (see Fig.1). If we define a non-dimensional friction variable $\tau$ as: 
$\tau=\frac{F_{T}}{\mu \lambda}$

The standard Coulomb friction condition then can be expressed as:

$|\tau| \leq 1$

And $|\tau|<1$ implies $\dot{u}=0$

$|\tau|=1$ implies $\operatorname{sign}(\dot{u})=\operatorname{sign}(\tau)$

Where $\dot{u}$ is the sliding velocity.

A multilinear frictional constraint function formed $\mathrm{v}(\dot{\mathrm{u}}, \tau)=0$ is used to regularize the friction, as shown in Fig.3, where $\varepsilon_{N}$ is a small parameter, and used only if friction is specified, the default value is $1.0 \times 10^{-3}$.

Thus, the possible states of the internal surface of steel tube and filled RC are: (i) the gap between the contactor nodes and/or segments of steel tube and the target segment of RC is closed; a compression force determined by Eq. (1) $(\mathrm{g}=0, \lambda>0)$ is acting onto the contactor nodes of steel tube and the nodes of steel tube kinematically slides along the target segments of RC with or without friction; (ii) the contactor nodes and/or segments of steel tube permanently attached or constrained to the target segment of RC, steel tube and $\mathrm{RC}$ then tied or bonded and deform together.

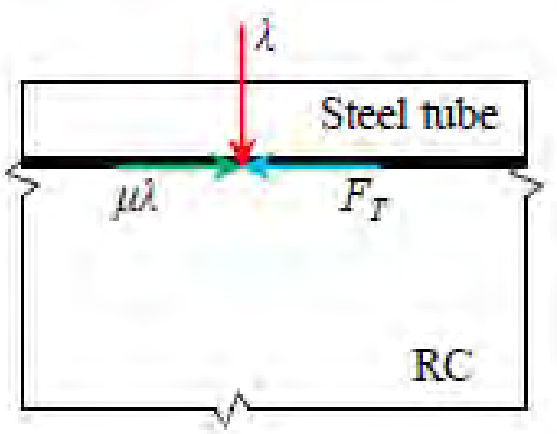

Fig. 1 Forces on Contact Surface

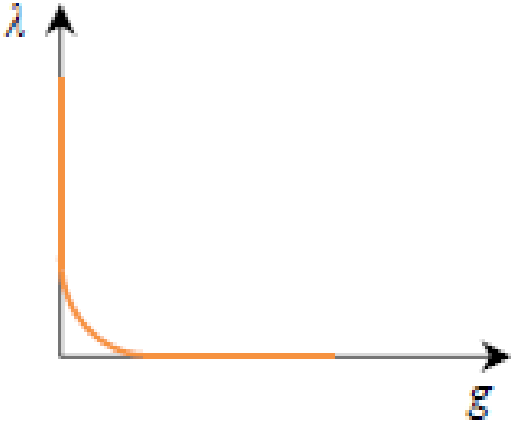

Fig. 2 Constraint Function

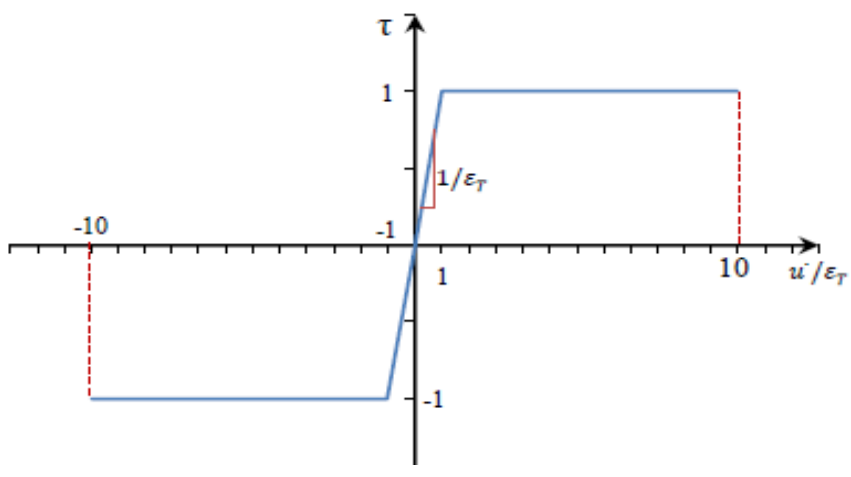

Fig. 3 Constraint Function for Frictional Contact

\section{Parameters for Simulation}

Corresponding to the selected three experimental RCFT columns, one frictionless, three frictional and one bonded conditions for each column total 15 different RCFT column models are determined based upon the conditions of former studies. The decided modeling parameters are listed in Table 2 . In the labels, the letter before "_" are labels of experimental RCFT columns, the "F" after "." refers to "Friction", number after "F" refers to decimal-point-removed coefficient of friction, "B" implies "Bond", "T" after "_" implies the results of test. 
Using the proposed material models for confined concrete, steel tube and reinforcement by Xiamuxi et al. [10], the determined RCFT models are simulated.

\section{Results of Simulation and Discussions}

The load-displacement curves of simulation are drawn in comparison with that of experiment for T45, T32 and T23 columns in Fig. 4.

To examine the agreement of the analytical curves against experimental curves, the correlation coefficients $\mathrm{R}^{2}$ of analytical curves are calculated against experimental curves and listed in Table 1 , Table 2and Table 3, respectively for T45, T32 and T23, including the comparable factors in numerical simulation such as memory usage, solution time and convergence.

(1)According to Table 4, the curves of boned condition are more close to the curves of experiment. Again, $\mathrm{R}^{2}$ of bonded conditions are greater than that of other conditions, which means that the simulation results of bonded condition are better agreement with experimental results than other conditions.

(2)No any clear difference can be observed between the curves of frictionless and all frictional conditions.

(3)As far as the memory usage is concerned, the frictionless conditions showed minimum usage (say 47.3MB, 49.6 MB and 48.3 MB) while the bonded conditions showed maximum usage (say 55.4MB, $58.5 \mathrm{MB}$ and 59.2 $\mathrm{MB}$ ), and there is no difference of memory usage between frictional conditions, which means that the frictionless contact consumes less computer resource compared with both frictional and bonded contact.

(4)As far as the solution time is concerned, corresponding to the memory usage, the frictionless conditions showed fastest solution (say $0.77 \mathrm{Sec}$., $0.91 \mathrm{Sec}$. and $0.80 \mathrm{Sec}$.) while the bonded conditions showed slowest solution (say $1.46 \mathrm{Sec}$., $1.54 \mathrm{Sec}$. and $1.75 \mathrm{Sec}$.), and solution time increases along with the increase of friction coefficient. The frictionless conditions are faster than bonded conditions almost one times, which means that the frictionless contacts are faster and most time-saving compared with both frictional and bonded contact.

(5)As far as the convergence is concerned, the boned conditions showed a little better convergence than other conditions.

(6)The minimum value of $\mathrm{R}^{2}$ among all columns is $\mathrm{R}^{2}=0.8611$ (with T23-F08), which means that all of the analytical results match experimental results well. Again, according to further observation on Fig.4, it can be noticed that the curves are in a good agreement until maximum load, and changes begin to happen between bonded and bond-less conditions only when larger plastic deformations started to happen, which mean that all of simulation results are under applicable range.

Tab. 3 Comparisons of Simulation Results with T45

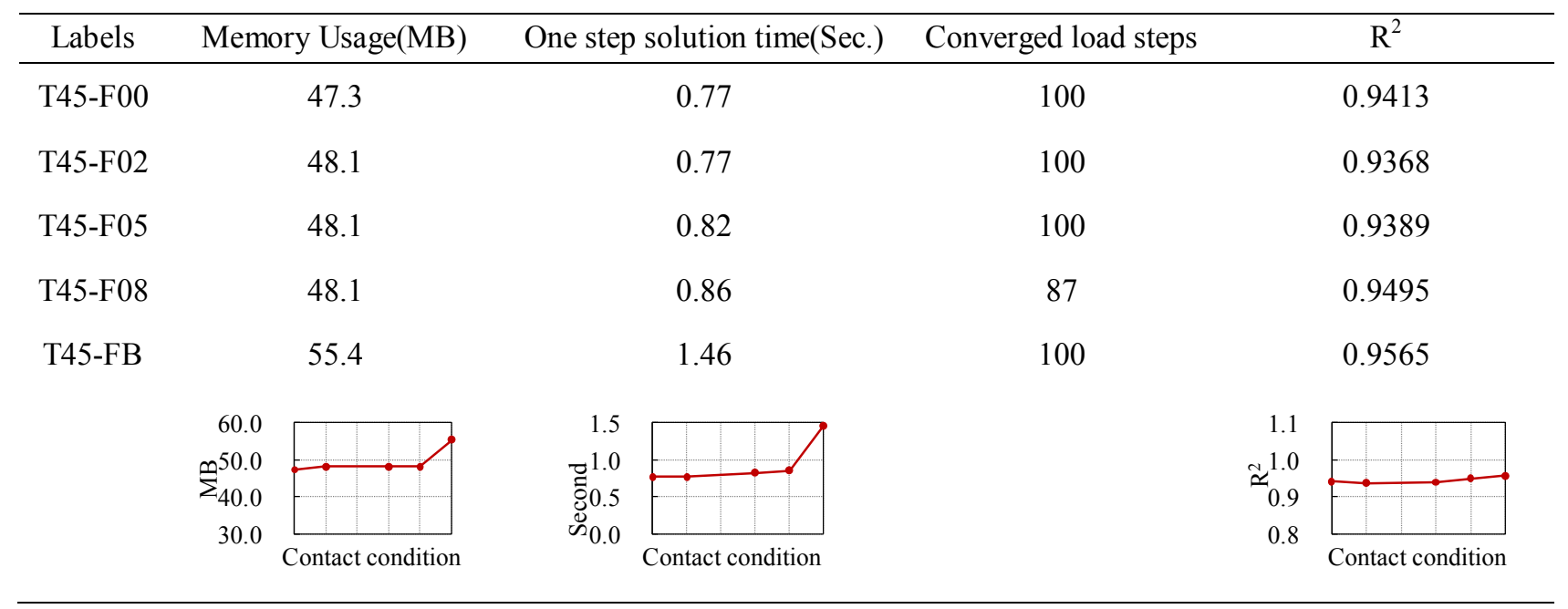


Tab. 4 Comparisons of Simulation Results with T32

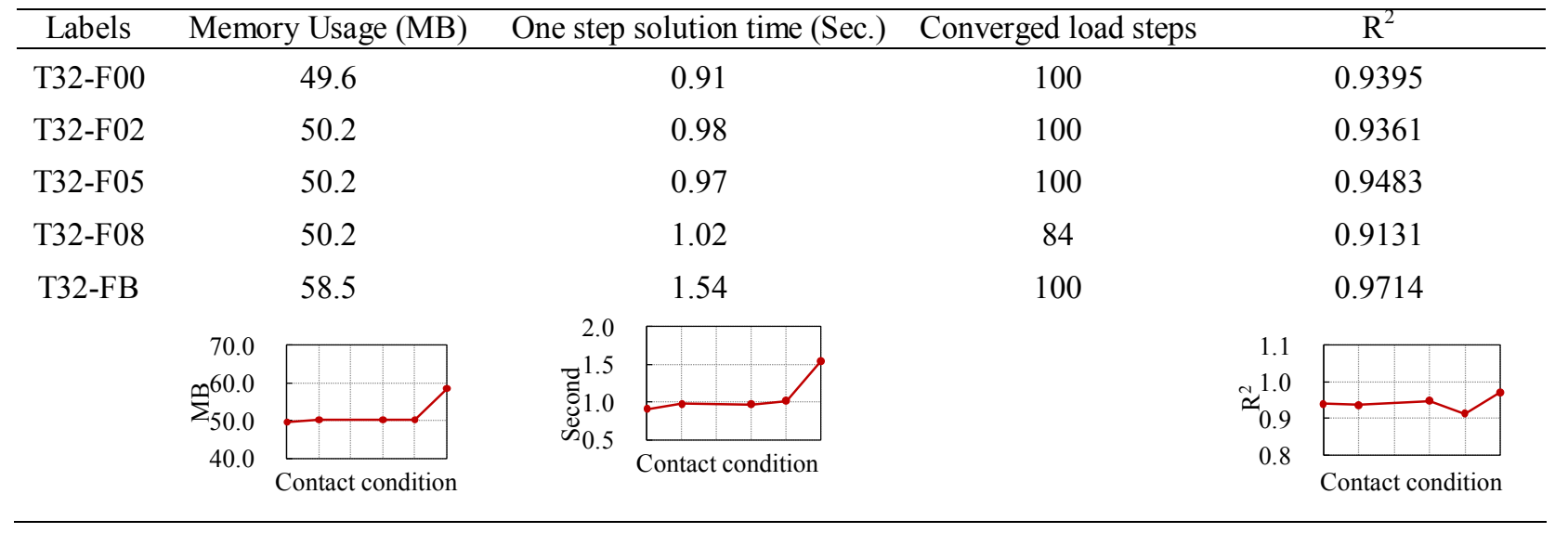

Tab. 5 Comparisons of Simulation Results with T23

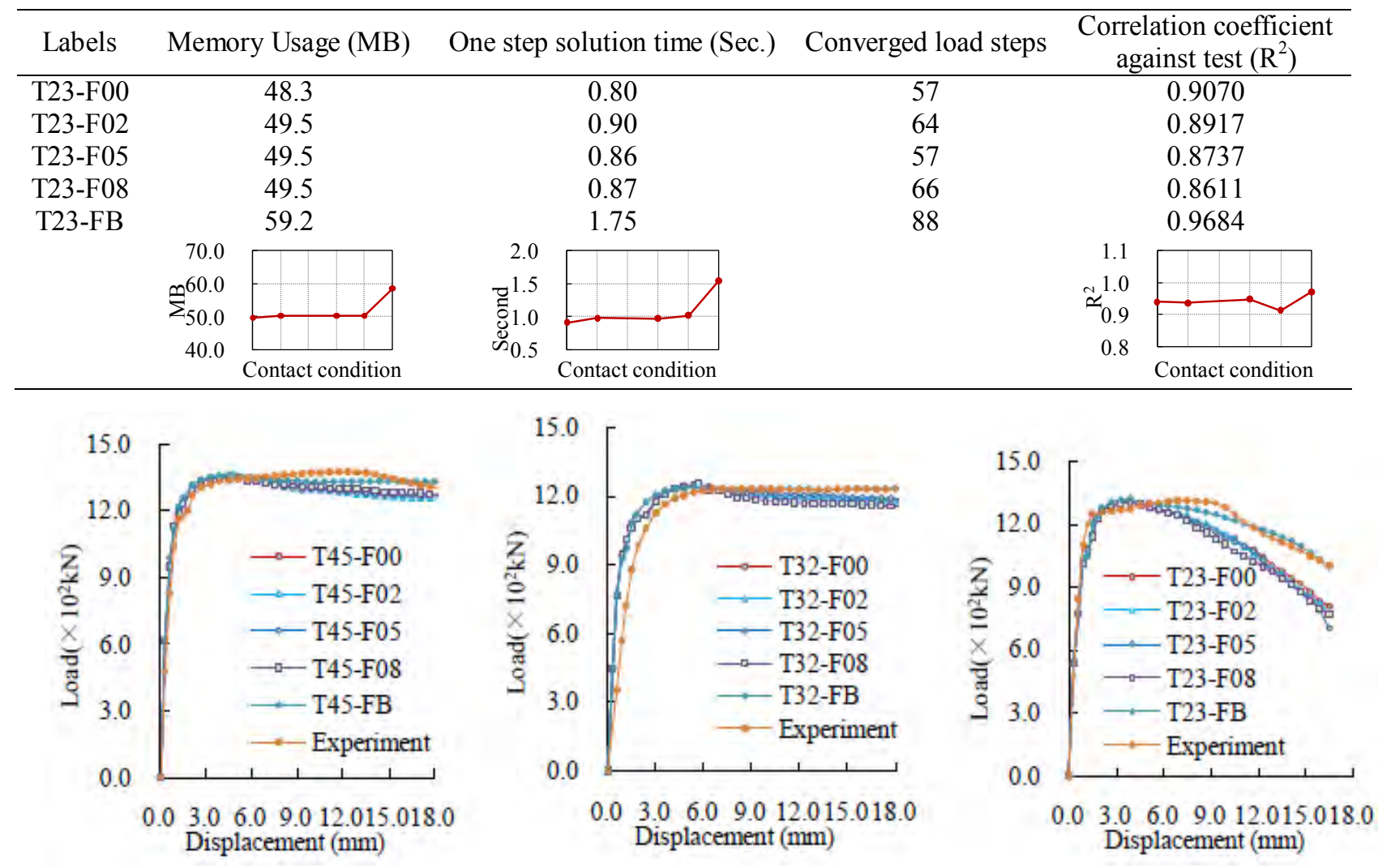

Fig. 4 Comparisons of Load-displacement Curves

\section{Summary}

Summarizing the discussions above, it can be concluded that when model the contact between steel tube and concrete in RCFT columns subjected axial compression, both bonded contact and frictional and frictionless contacts are applicable. Bonded contact may be recommended to obtain more accurate results if time and computer resource is not concerned. On the contrary, the frictionless contact is recommended to obtain faster solutions.

\section{Acknowledgement}

The research described in this paper was financially supported by the Key Projects of Regional 
Education Department of Xinjiang (XJEDU2014I006), the Starting-up Research Foundation of Xinjiang University for Doctors (BS130125), the Scientific Research Foundation for the Returned Overseas Chinese Scholars, State Education Ministry and the Regional Academic Discipline (Breeding) Program for "Structural Engineering", Xinjiang.

\section{References}

[1]K.K. Choi and X. Yan: Analytical model of circular CFRP confined concrete-filled steel tubular columns under axial compression, Journal of Composites for Construction, ASCE, Vol.14, No.1, pp.125-128, 2010.1.

[2]H.T. Hu, C.S. Huang, M.H. Wu et al.: Nonlinear analysis of axially loaded concrete-filled tube columns with confinement effect, Journal of Structural Engineering, ASCE, Vol.129 No.10, pp.1322-1329, 2003.10.

[3]W. Li, L. H. Han and X.L. Zhao: Numerical investigation of concrete filled double skin tubular (CFDST) columns with preloads on steel tubes, The 2011 World Congress on Advances in Structural Engineering and Mechanics (ASEM'11 ${ }^{\dagger}$ ), Seoul Korea, pp.2345-2352, 2011.9.

[4]L.H. Han, G. H. Yao and Z. Tao: Performance of concrete-filled thin-walled steel tubes under pure torsion, Thin-Walled Structures, Vol.45, No.1, pp.24-36, 2007.1.

[5]Y. Zeng, C.C.Y. Leung and F.T.K. Au: Finite element analysis of in-situ stitches in precast concrete segmental bridges, The 2011 World Congress on Advances in Structural Engineering and Mechanics (ASEM'11 ${ }^{+}$), Seoul Korea, pp.1563-1572, 2011.9.

[6]C. C. Hou, L. H. Han and Z. Tao: Simulation on concrete-filled steel tubular members under transverse impact, The 2011 World Congress on Advances in Structural Engineering and Mechanics (ASEM'11 ${ }^{+}$), Seoul Korea, pp.4189-4196, 2011.9.

[7]P.F. Mao, D.L. Wang: Nonlinear finite element modeling of the interior steel-concrete composite beam joints, International Journal of Nonlinear Science, Vol.11, No.2, pp.173-179, 2011.4.

[8]H.G. Kwak, J. H. Kwak and H.-G. Gang: Cyclic behavior of circular concrete filled steel tubular column, The 2011 World Congress on Advances in Structural Engineering and Mechanics (ASEM'11 ${ }^{+}$), Seoul Korea, pp.1476-1484, 2011.9.

[9]T. Suzuki: Study on new bridges that adopt hybrid structure, PhD thesis, Hachinohe Institute of Technology, Hachinohe Japan, 2008.2.

[10]A. Xiamuxi and A. Hasegawa: A study on axial compressive behaviors of reinforced concrete filled tubular steel columns, Journal of Constructional Steel Research, JCSR, Vol. 76, pp. 144-154, 2012.9 . 